Original paper

\title{
Radiomics software for breast imaging optimization and simulation studies
}

\author{
Stoyko Marinov ${ }^{\mathrm{c}}$, Ivan Buliev ${ }^{\mathrm{b}}$, Lesley Cockmartin ${ }^{\mathrm{a}}$, Hilde Bosmans ${ }^{\mathrm{a}, \mathrm{c}}$, Zhivko Bliznakov ${ }^{\mathrm{d}}$, \\ Giovanni Mettivier ${ }^{\mathrm{e}}$, Paolo Russo ${ }^{\mathrm{e}}$, Kristina Bliznakova ${ }^{\mathrm{d}, *}$ \\ ${ }^{a}$ Department of Radiology, University Hospitals Leuven, Herestraat 49, 3000 Leuven, Belgium \\ ${ }^{\mathrm{b}}$ Mikrosistemi Ltd., Varna, Bulgaria \\ ${ }^{\mathrm{c}}$ Medical Physics and Quality Assessment, Department of Imaging and Pathology, KU Leuven, Herestraat 49, 3000 Leuven, Belgium \\ ${ }^{\mathrm{d}}$ Department of Medical Equipment, Electronic and Information Technologies in Healthcare, Medical University of Varna, Varna, Bulgaria \\ e Dipartimento di Fisica "Ettore Pancini", Universita' di Napoli Federico II and INFN Sezione di Napoli, Naples, Italy
}

\section{A R T I C L E I N F O}

\section{Keywords:}

Evaluation of $\mathrm{x}$-ray images

Breast images

Statistical features

Fractal dimension

Power law analysis

Matrix analyses

\begin{abstract}
A B S T R A C T
Background and Objective: The development, control and optimisation of new x-ray breast imaging modalities could benefit from a quantitative assessment of the resulting image textures. The aim of this work was to develop a software tool for routine radiomics applications in breast imaging, which will also be available upon request. Methods: The tool (developed in MATLAB) allows image reading, selection of Regions of Interest (ROI), analysis and comparison. Requirements towards the tool also included convenient handling of common medical and simulated images, building and providing a library of commonly applied algorithms and a friendly graphical user interface. Initial set of features and analyses have been selected after a literature search. Being open, the tool can be extended, if necessary.

Results: The tool allows semi-automatic extracting of ROIs, calculating and processing a total of 23 different metrics or features in 2D images and/or in 3D image volumes. Computations of the features were verified against computations with other software packages performed with test images. Two case studies illustrate the applicability of the tool - (i) features on a series of 2D 'left' and 'right' CC mammograms acquired on a Siemens Inspiration system were computed and compared, and (ii) evaluation of the suitability of newly proposed and developed breast phantoms for x-ray-based imaging based on reference values from clinical mammography images. Obtained results could steer the further development of the physical breast phantoms.

Conclusions: A new image analysis toolbox was realized and can now be used in a multitude of radiomics applications, on both clinical and test images.
\end{abstract}

\section{Introduction}

Ionizing radiation is routinely used in medical diagnosis of the breast: x-ray acquisitions are the basis of all radiological modalities including Full-Field Digital Mammography and Breast Tomosynthesis. These modalities are improved in a continuous effort, with better x-ray sources and detectors, acquisition geometry, reconstruction algorithms, etc. Modelling and simulation play an important role in this process, by providing a potentially less expensive alternative to the experimental approach that involves the manufacturing of prototypes, access to patient cohorts and expensive processing of data [1-10]. Regardless of the chosen approach, improvement of existing imaging techniques and the development of new ones such as dedicated breast CT [11-16], phase contrast breast CT [17-19] or dual energy CT [20] require phases of validation and extensive testing.

Validation of any new evolution in breast x-ray imaging is in many cases related to an objective evaluation, based on different features and figures of merit (FOMs), computed from the images. Depending on the clinical imaging task to be optimized, specific metrics are available. While improved detectability of lesion-like signals is often an end goal, expressed with metrics such as signal difference-to-noise ratio and threshold contrast, our current project addresses applications that may benefit from differences in texture of the image. For example, in digital mammography, the complexity of the mammograms has been described with parameters such as the $\beta$ value of the power spectrum envelope in the spatial frequency domain $[5,21-24]$. Other parameters include the fractal dimension [25], used to classify the breast parenchyma as very dense, scattered glandular tissue or fatty [26-30]. Some of these features

\footnotetext{
* Corresponding author.

E-mail address: kristina.bliznakova@mu-varna.bg (K. Bliznakova).
} 

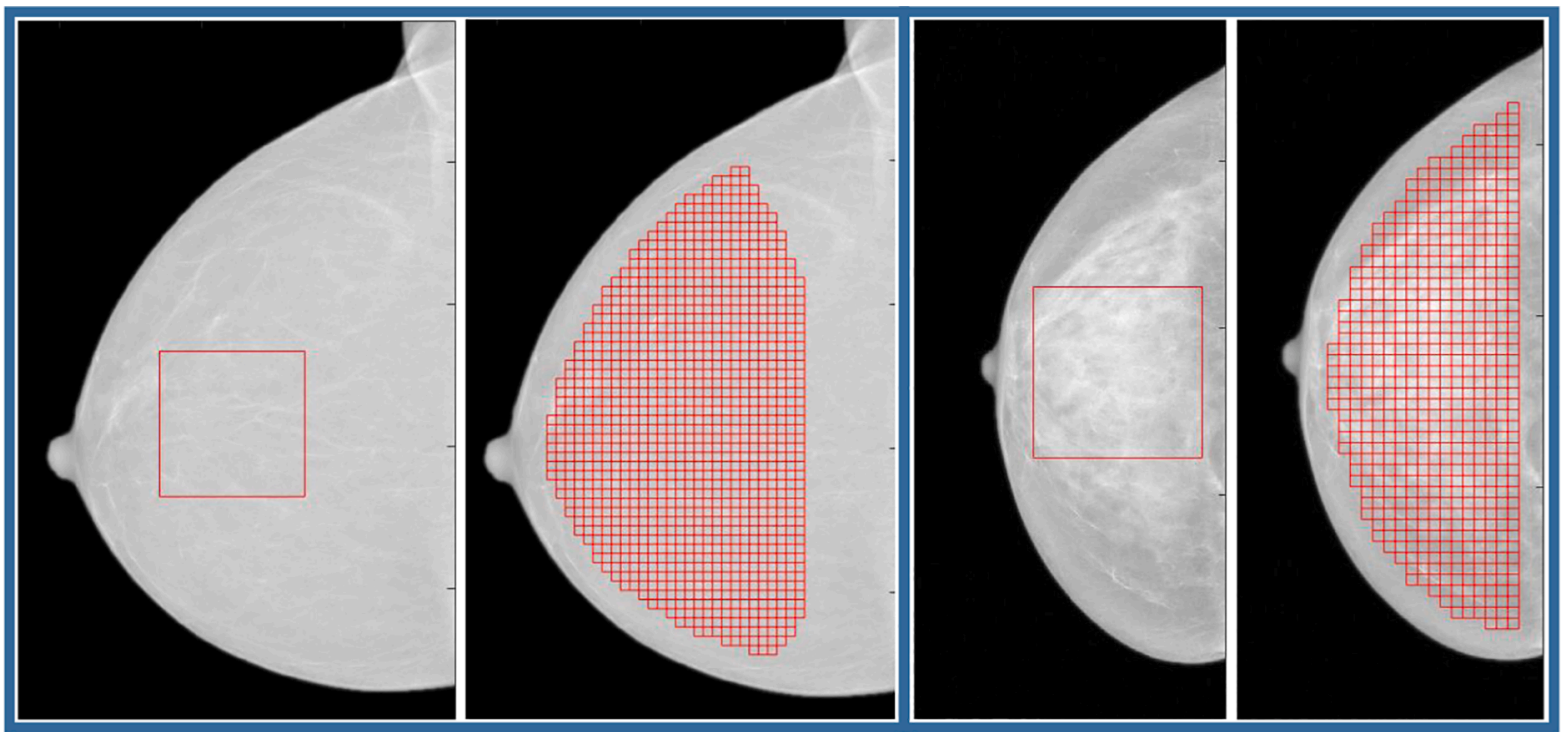

Fractal Dimension $=3.15 ;$ Skewness $=0.23 \pm 0.27 ;$ Kurtosis $=3.07 \pm 0.56$

Fractal Dimension $=2.93 ;$ Skewness $=0.12 \pm 0.38 ;$ Kurtosis $=2.89 \pm 0.71$

(a)

(b)

Fig. 1. Examples for feature extraction through ROIs from (a) fatty and (b) glandular breasts. Skewness and kurtosis are measured by using ROIs of $32 \times 32$ pixels, while the fractal dimension and power spectrum analysis are measured by using a single ROI of $512 \times 512$ pixels.

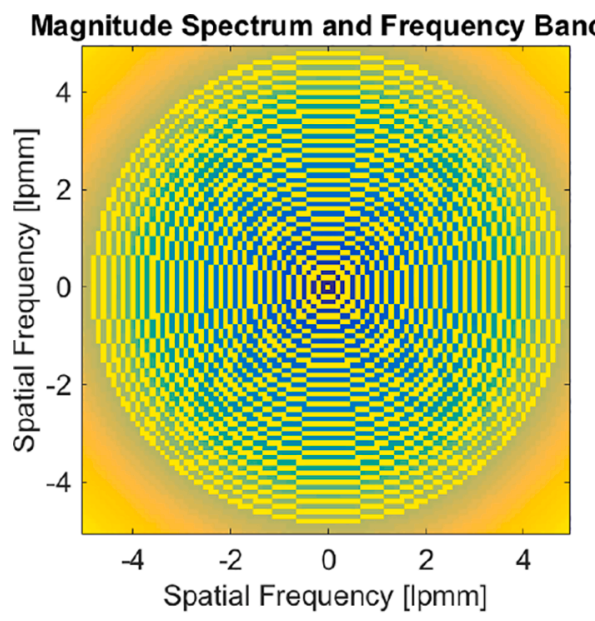

(a)

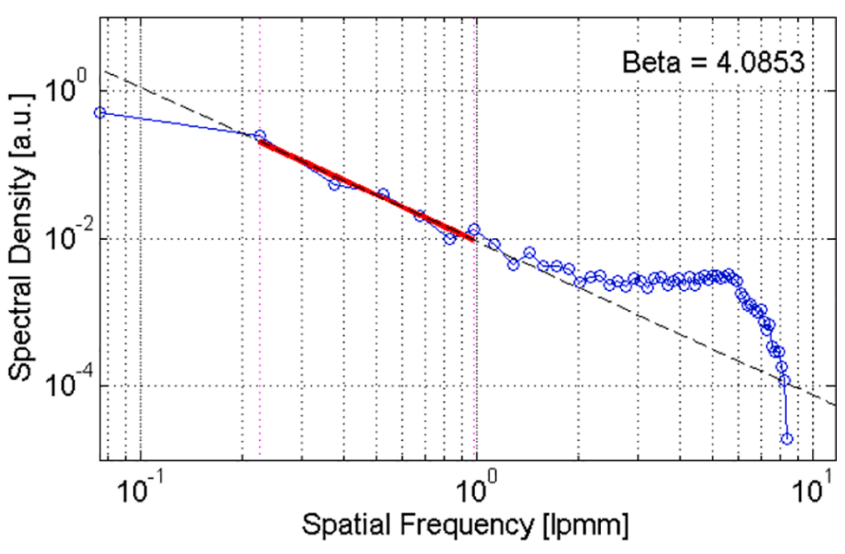

(b)

Fig. 2. ROI spectrum and superimposed rings (in yellow) of linearly distributed frequency bands with every 2nd ring omitted for visual clarity (a), and the power spectra of a ROI of $128 \times 128$ pixels taken from the central part of the mammography image. (For interpretation of the references to colour in this figure legend, the reader is referred to the web version of this article.)

are also known to correlate with breast cancer risk analysis [31-34]. Optimization of the imaging chain may aim to detect specific patterns or textures, to preserve them or to improve specific aspects. Textures can be particularly influenced when switching to 3D imaging modalities, at the implementation of subtraction techniques, with multi modal imaging or artificial intelligence-based processing or reconstruction.

The aim of this work was to build up a radiomics toolbox to quantify mammographic textures, to track feature changes in comparison to base line and/or study their impact on performance. Features to be implemented were first selected from literature, and then further expanded with possibly interesting extra functions using MATLAB coding. The developed toolbox is intended to facilitate validation and evaluation studies performed by researchers and to enable easy (routine) quality control measurements (of breast texture) by clinical medical physicists.
In the following, we illustrate typical results from two state-of-the-art applications.

The following requirements were put forward: (i) the toolbox should address the whole spectrum of 2D and 3D breast imaging techniques; (ii) common features defined in the literature should be included, (iii) the algorithms have to be validated, against computations with other software packages, and (iv) the (graphical) user interface (GUI) has to be intuitive, attractive and time-efficient for common applications. Therefore, the toolbox should offer the possibility to process different types of images, to manually or semi-automatically select regions of interest (ROIs), to compute a list of high-order statistics, spectral density and fractal dimension, and others, and adopt other potentially useful ones, such as the Laplacian fractional entropy [22] or any other promising function, with provision to save and/or print the output of the 


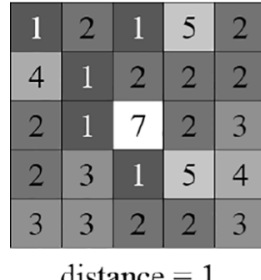

distance $=1$

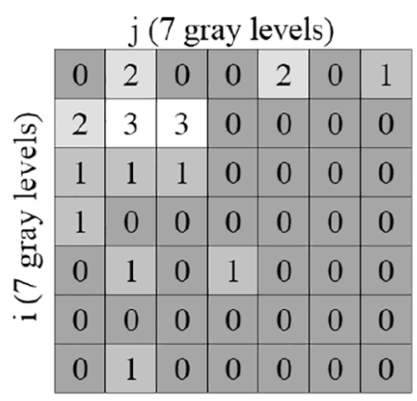

(b) $\mathrm{M}_{\mathrm{m}+}=\rightarrow$

(left to right)

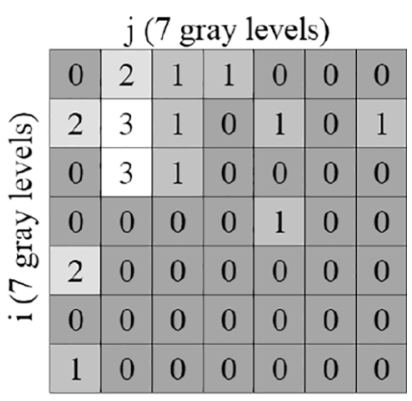

(c) $\mathrm{M}_{\mathrm{m}-}=\leftarrow$

(right to left)

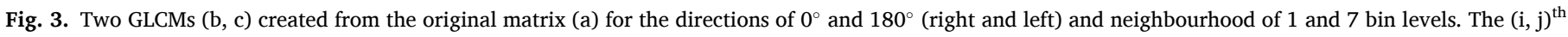
value in the matrices $(b, c)$ corresponds to the sum of the pairs with grey levels $i$ and $j$ encountered along the specified direction [36].

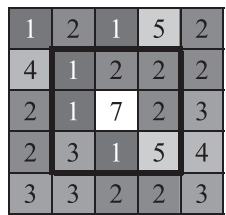

neighbourhood $=1$

\begin{tabular}{|c|c|c|c|}
\hline Gray Levels & $\mathrm{n}_{\mathrm{i}}$ & $\mathrm{p}_{\mathrm{i}}$ & $\mathrm{S}_{\mathrm{i}}$ \\
\hline 1 & 3 & 0.333 & 5.375 \\
\hline 2 & 3 & 0.333 & 2.875 \\
\hline 3 & 1 & 0.111 & 0.375 \\
\hline 4 & 0 & 0.000 & 0 \\
\hline 5 & 1 & 0.111 & 2 \\
\hline 6 & 0 & 0.000 & 0 \\
\hline 7 & 1 & 0.111 & 4.875 \\
\hline
\end{tabular}
(b) Neighbourhood Gray
Level Difference Matrix
(a) gray levels of
an example matrix

radiomics features, which helps for validating the feature computation by different tools. The implemented feature set in our applications is not frozen - it is rather subject to further extension by the group, but can also be enriched by any other user.

In the current implementation, calculations are performed for every individual region of interest (ROI) in the studied image. ROIs in the tool can be created by following two approaches: (i) manually, by placing each ROI by hand and (ii) semi-automatically, by configuring a region for placement in the image, overlapping method and ROI size. The final reported result for the features is an average of the results from these individual regions of interest (ROIs). The following subsections describe the theoretical basis of the implemented features. For clarity, the mathematical equations, marked with the letter $\mathrm{A}$, are provided in the Appendix Part A.

\subsection{Histogram analysis}

For each ROI, the tool computes the following statistics: probability of a pixel/voxel being of intensity level $i, \mathrm{~s}_{i}$ is the neighbourhood grey level difference for pixels/voxels with grey level $i$.

processing. Moreover, definition of baseline values, reference data and mutual comparisons were considered standard output.

Such a toolbox can be set up in different ways. There exist software applications to load images from different modalities and extract some general-purpose image features. One can also find and use dedicated scripts for popular image processing tools like ImageJ, MATLAB, IDL and even radiomics packages such as pyRadiomics [35]. However, for some specific applications, a dedicated toolbox would be much more valuable, also taking into account the increasing amount of big data applications, and the availability of large data sets for artificial intelligence (AI) and neural networks and other evaluations. Radiomics may be useful for data cleaning prior to any further (AI) application and to acquire 'explainable' data compared to neural network outputs, to add data to training parameters, or as a next or parallel evaluation. All this is only possible with appropriate processing tools. We present two applications of the toolbox: evaluation of features from a set of 'left' and 'right' craniocaudal (CC) mammograms and evaluation of the suitability of newly proposed and developed breast phantoms for x-ray-based imaging based on reference values from clinical mammography images.

\section{Materials and methods}

The features currently included in the toolbox are often used for analysis of mammographic images as reported in literature. Most features are referenced from the IBSI (Image Biomarker Standardization Initiative) document, created by groups of researchers [36]. The IBSI document provides standardized definitions for a large number of
- Mean value and standard deviation of the intensities (pixel values) across the ROIs.

- Skewness, i.e., the distortion or asymmetry of the data distribution compared to the normal bell curve. The symmetry of the frequency distribution of the values around the mean value is considered informative in breast image analysis, e.g., tailing in the pixel value histogram might be due to microcalcifications. Also, skewness values are computed from small ROIs and then averaged over the whole image. The resulting value could be used to determine the glandularity of a breast to some extent, as stated by Byng et al. [26]. Specifically, for a distribution, which tends toward lower grey levels, i.e., a region of fatty tissue, a positive skewness is expected, while for dense breast parts a negative skewness is expected. A commonly used computation, following equation A1 in the Appendix, is implemented in the tool.

- Kurtosis, measures the combined weight of the tails, relative to the rest of the distribution [37]. The expression used in the tool, is described by equation A2. In case of mammography imaging, outliers of the intensity, such as microcalcification, result in higher or lower values of the kurtosis, depending on the relative sizes of the microcalcification and the ROI. Both skewness and kurtosis were used by Bochud et al. [37] to validate quantitatively the realism of the generated synthetic mammograms against patient images.

\subsection{Fractal analysis}

The fractal dimension of the images correlates with the roughness of the observed textured areas. Higher fractal dimension corresponds to rougher textures and vice versa and can be used for image 


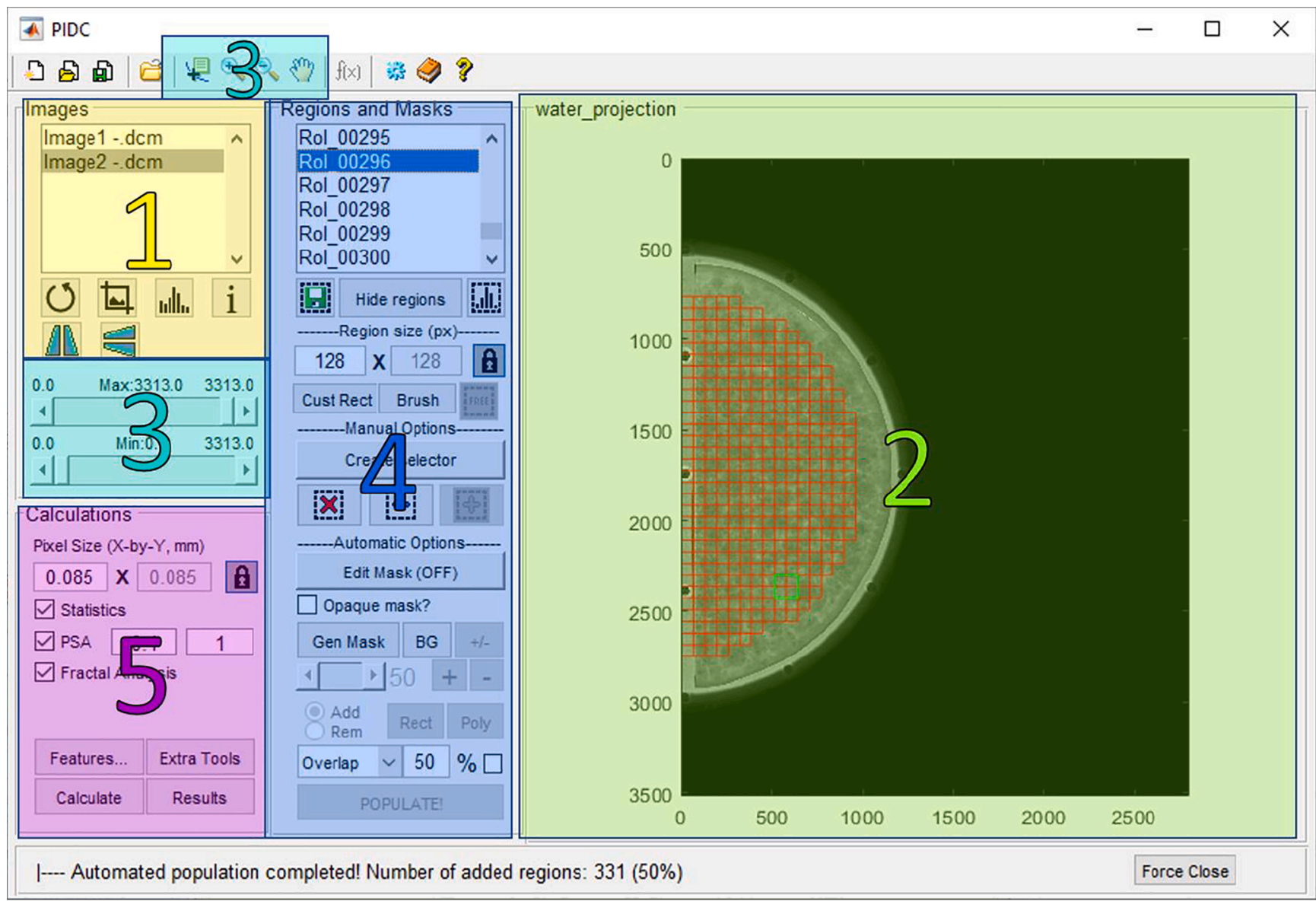

Fig. 5. A screenshot of the main window of the tool - the numbered areas depict the tools dedicated to certain task.

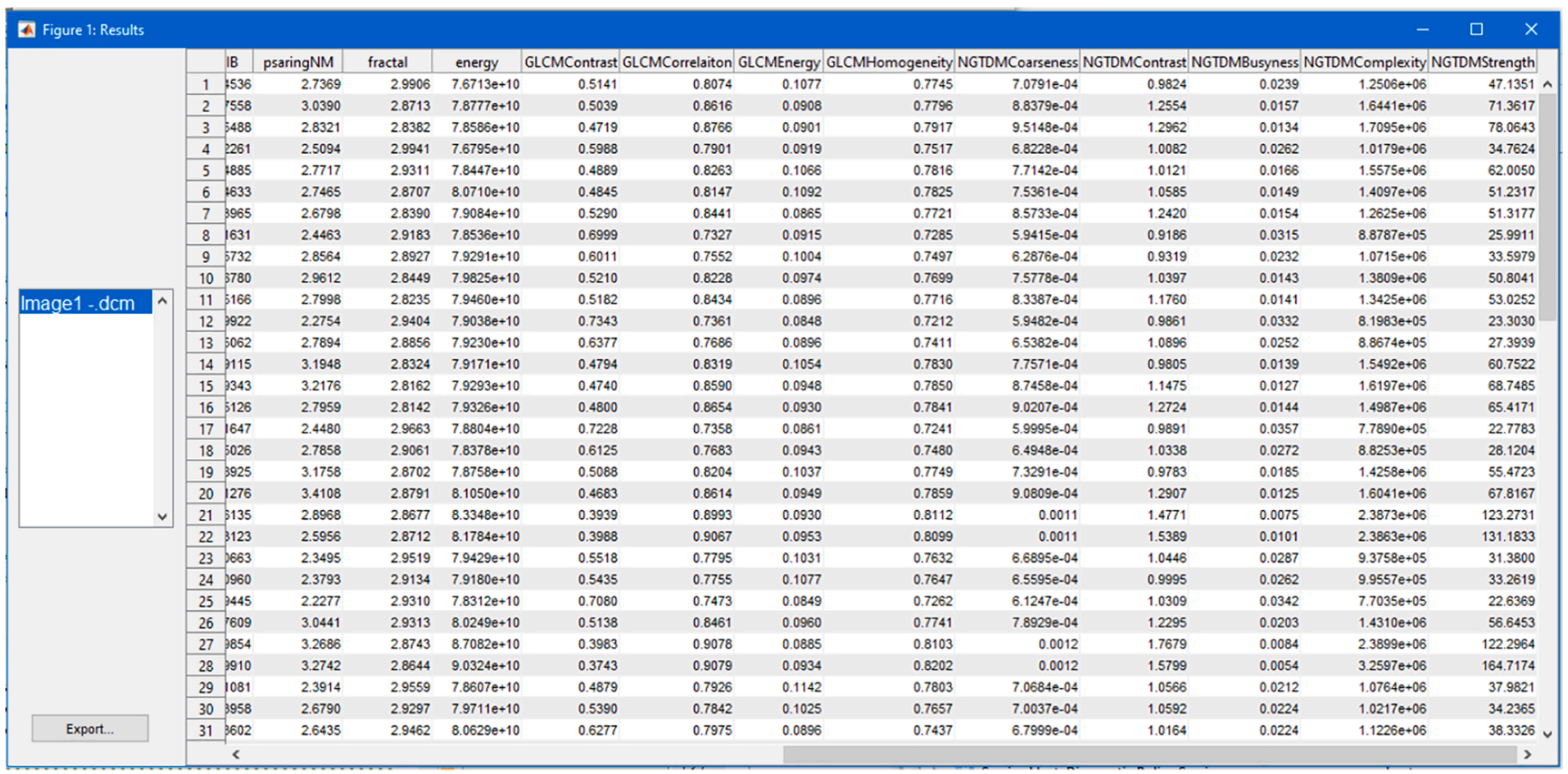

Fig. 6. Results window for the calculated features for each ROI in the mammography image. 


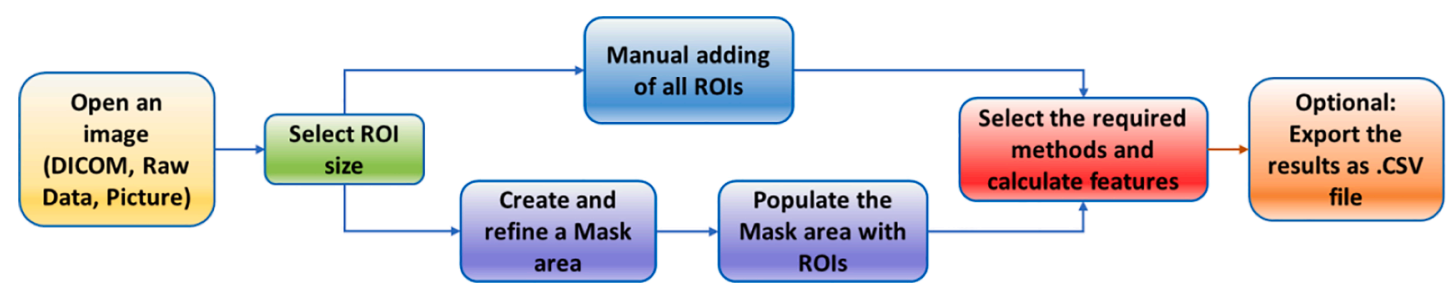

Fig. 7. A flowchart of the common usage of the tool.

characterization. In the case of mammography, it may be used as an indicator of the breast density [21]. There exist several methods for calculating the fractal dimension [38]. The one currently implemented in the tool is the "box-counting technique" described by Byng et al. 1996 [26], shown by equation A3. Li et al. [39] demonstrated that the fractal dimension may be used as an indicator to assess the breast parenchyma, and thus inherently includes a prediction for low or high breast cancer risk. Example features extracted from the histogram of the mammography images of a fatty (ACR1) and a glandular (ACR4) breast are shown in Fig. 1. Two types of ROIs differing in their size $-32 \times 32$ pixels (3.2 $\mathrm{mm} \times 3.2 \mathrm{~mm})$ and $512 \times 512$ pixels $(51.2 \mathrm{~mm} \times 51.2 \mathrm{~mm})-$ were used to evaluate the features. Skewness and kurtosis were calculated based on the smaller ROIs, since they reflect the local texture of the finer details. Similarly, to the results of Byng et al. [26] and Li et al. [40], fractal dimension and skewness values of mammography images of a fatty breast are higher (Fig. 1a) compared to the values of these parameters extracted from the mammography image of a dense breast (Fig. 1b).

\subsection{Spectral analysis and power law}

The power spectrum describes the strength of the spatial frequencies present in the image. For mammographic images, the magnitude of the low-frequency components shows a power law [41] as described in A4 in the Appendix.

The tool computes the centred version of the spectral density of the image, in which the frequency bands of interest are represented by central concentric rings of corresponding width, as shown in Fig. 2a. The frequency bands can be integrated or averaged. For calculation of $\beta$, the logarithm to the base 2 of the total energy per ring is then plotted versus the logarithm of the central frequencies of the bands (Fig. 2b).

\subsection{Grey level Co-occurrence matrix (GLCM)}

GLCM, first defined by Haralick et al. [42], is used to calculate features from an image such as texture, structural patterns and their spatial relationship. The method creates a matrix that expresses how combinations of pairs of discretised grey levels (intensities) in specified neighbourhoods are distributed along one or more of the image directions (left to right, bottom to top, etc., Fig. 3). These features rely on the probability distribution of the elements in the GLCM that are created by normalizing the GLCM matrix and integrating along the rows, columns and diagonals [36].

The IBSI document contains 25 possible features for calculation from the GLCM matrix. The descriptions of the features are referenced from the IBSI document [36]. Based on those features, Li et al. [40] showed that gene mutation carriers and low-risk women have different mammographic patterns. The mammographic images for the gene have coarse texture, which is indicated by high coarseness values, low fractal dimension and low entropy. Features were also used to classify breast textural patterns and assess the breast cancer risk [39,43-45].

\section{- GLCM Energy (Also known as: Angular Second Momentum, Uniformity)}

The GLCM Energy is also known as uniformity, uniformity of energy, or angular second moment, and is calculated as described by equation A5 in the Appendix. This descriptor is used for evaluation of the structural patterns throughout the whole texture.

\section{- GLCM Contrast (Also known as: Variance, Inertia)}

The GLCM Contrast feature adds the differences in the intensity between a pixel and its neighbour for all pixels in an ROI. This means that the greater the variation, the greater the final value will be. GLCM Contrast is calculated by using equation A6. This descriptor is used for evaluation of the local variations throughout the texture.

\section{- GLCM Correlation}

The GLCM Correlation feature measures how correlated a specific pixel is to its neighbouring pixels over the whole image. The correlation is calculated by using equation A7.

\section{- GLCM Homogeneity (Also known as: Inverse Difference)}

The GLCM Homogeneity measures the closeness of the distribution of the elements in the GLCM to the diagonal. This feature is calculated by using equation $\mathrm{A} 8$.

\subsection{Neighbourhood grey tone difference matrix (NGTDM)}

This feature extraction method was introduced by Amadasun and King [46] as an alternative to the GLCM. The main difference is that the NGTDM does not look at the pixel pairs in specific directions, but at the closest neighbouring pixels/voxels up to a specified Chebishev distance.

The NGTDM matrix can be calculated in two ways - by using only the valid neighbourhoods ( 8 for a 2D image and 26 for a 3D image, with Chebishev distance of 1 ), which is the original definition given by Adamasun and King [46], or by using the incomplete neighbourhood the latter method is an extended/improved method introduced in the IBSI document. In the example in Fig. 4a, all pixels, which do have valid neighbourhoods and on which a feature matrix can be calculated, are outlined. The pixels outside the boxed area will be ignored. Both methods are available for calculation in the toolbox. The calculated features from the resulting NGTDM matrix are Coarseness, Contrast, Busyness, Complexity and Strength. The reference and the description of the features are derived from the IBSI document. The features are used in evaluating breast textural patterns aiming at the breast risk prediction $[40,43]$. 


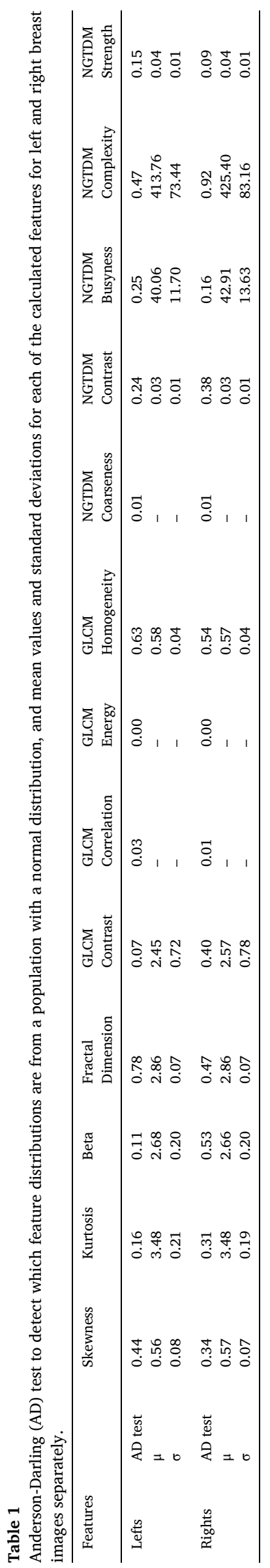

- NGTDM Coarseness: The NGTDM Coarseness feature indicates the level of the spatial rate of change in intensity and is calculated by equation A9.

- NGTDM Contrast: The NGTDM Contrast feature depends on the dynamic range of the intensity levels as well as the spatial frequency of intensity changes. It is calculated by using equation A10.

- NGTDM Busyness: The NGTDM Busyness feature describes the changes in the intensity between neighbouring pixels. Large changes are defining the image as busy. This feature is calculated by equation A11.

- NGTDM Complexity: The NGTDM Complexity feature describes how common are the non-uniform and rapid changes in the grey levels. It is calculated by equation A12.

- NGTDM Strength: The NGTDM Strength of the texture is defined by equation A13.

\subsection{Software environment}

The tool was implemented in MATLAB. Most of the computations were preliminary performed and tested in MATLAB. The coding of all features, except the fractal dimension, the power spectrum analysis and the statistical features, was verified against the data available in the IBSI document [36]. The coding of the statistical features was validated versus computations performed with the pyRadiomics library [35], while the power spectrum analysis was validated by replicating experimental results using the IDL code published by Marshall et al. [47]. The detailed validation of the features is presented in the Appendix Part $B$.

\section{Results and discussion}

The results of this work were separated in two categories. Firstly, we presented the user interface, image region selection and the practical usage of the tool. Secondly, we apply the tool to two research case studies in digital mammography.

\subsection{The toolbox and its GUI}

\section{- User interface}

Figure 5 shows the main screen of the user interface application of the toolbox. The user interface is implemented in MATLAB 2013a environment using the GUIDE module [48]. Consequently, the toolbox was upgraded to work also with MATLAB version 2020a, while at the same time keeping backwards compatibility. The five regions highlighted in this graphical interface relate to different activities and functionalities:

Region 1: Importing images: DICOM, raw data (no header) and other common picture formats (e.g., TIF, PNG, etc.) can be imported into the "Images" list. Particular raw data formats such as .NRRD and .NII are not supported at the moment. The current workspace (loaded images, performed analyses, defined ROIs) can also be saved and loaded. The four buttons under the list with image files in region 1 ("Images" list) allow reloading of the original image, cropping, computing the histogram, and obtaining additional information (displaying DICOM header) of the studied image. In the current toolbox version, the option to load and extract 3D image features (from 3D volume) is implemented in the form of separate scripts. Features extracted from tomography slices are evaluated in a similar way as 2D planar images.

Region 2: Visualization area, i.e., an area for displaying the loaded images. The image selected from the "Images" list is displayed on the right side in grayscale, preserving the original image aspect ratio.

Region 3: Tools for image inspection and control of the contrast. The sliders provided on the left allow contrast adjustments to be applied to the displayed image (Fig. 5, region 3). The toolbar additionally provides the user with controls to zoom in and out, to pan the image and to examine the data of a specific pixel. 

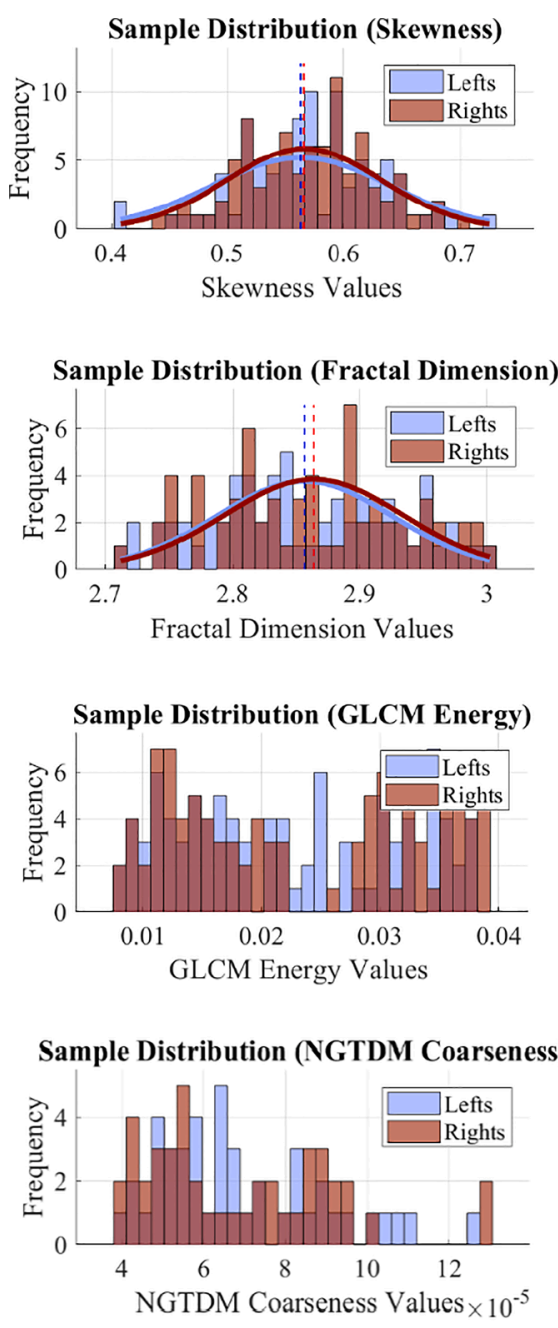
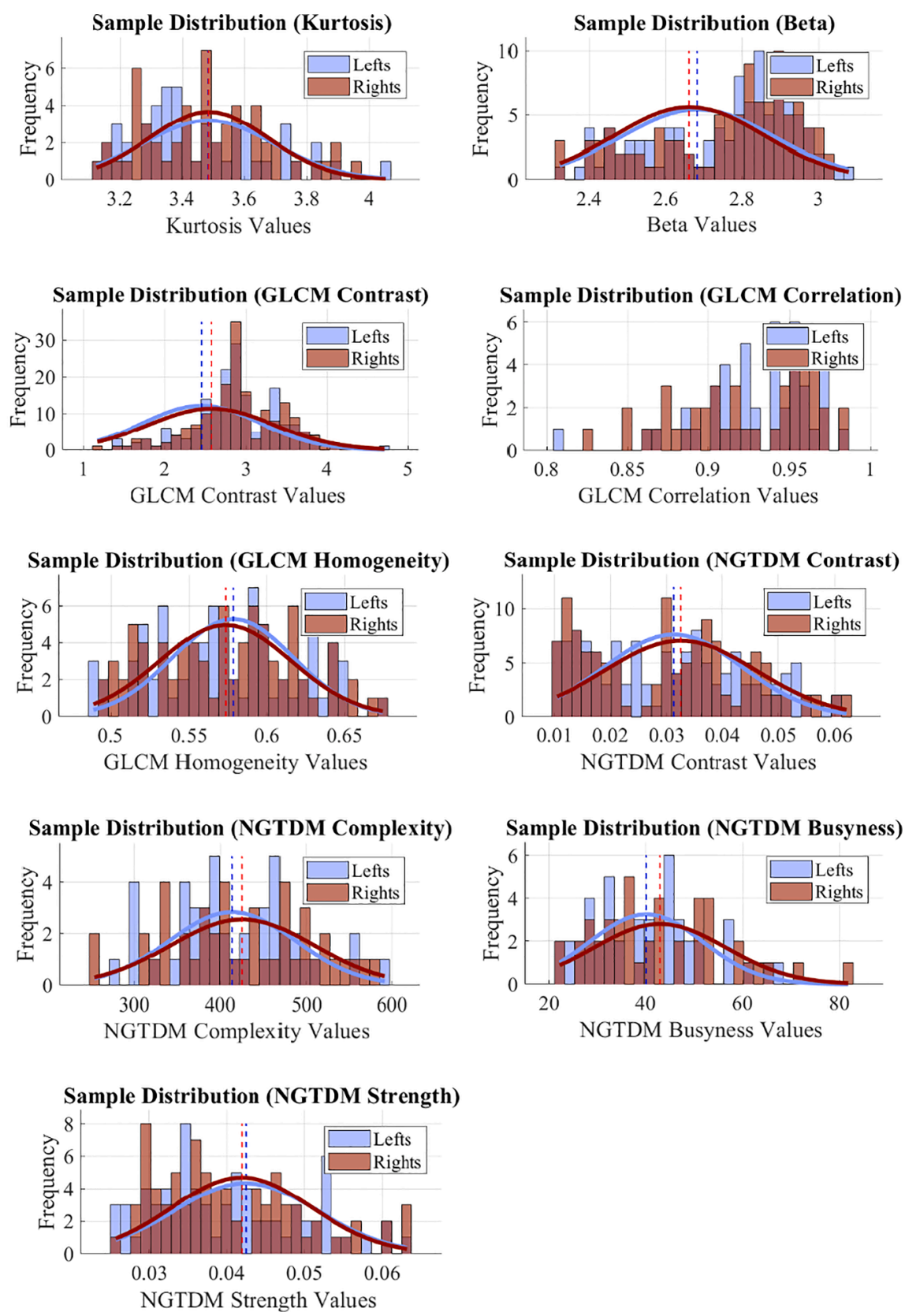

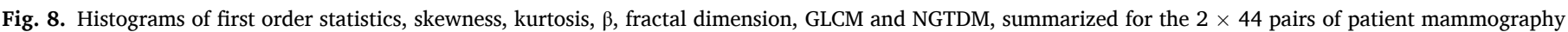
images. Gaussian fit is shown for the cases with the Gaussian distribution. 'Lefts' and 'Rights' stand for left CC and right CC mammography image, respectively.

Region 4: Definition of Regions of Interest. A group of controls (Fig. 5, region 4) is used to, manually or semi-automatically, create a mask and define ROIs in the selected image. Masks are binary matrices with the same dimensions as the loaded mammography image, the true values of which show the ROIs. The size and sampling method of the ROIs (either by percentage overlap or randomly) is selected by the user.

Region 5: Selection of FOMs. The user can select either a subset or the whole set of features from the 'Calculations' section (Fig. 5, region 5). Before performing calculations on the image, the user is required to add at least one ROI in the "Regions and Masks" list (Fig. 5, region 3). An example output of the calculations is shown in Fig. 6, where some of the calculated features are shown for the selected 289 ROIs. For some features, like the power spectrum analysis, additional information such as spectral density and the selected frequency range (Fig. 2b) are displayed. The titles of the columns follow the names of the features (Fig. 6). The calculations can be exported as a Comma Separated Value (CSV) file and used for further analysis and processing in other environments. For each feature, the corresponding histogram can be built based on the ROIs in the image and shown in separate windows. The latter can be saved and used into reports.

\section{- ROI selection tools}

The toolbox offers several tools for selection of the ROIs within the image, as depicted in Fig. 5 (area 4). First, the ROI dimensions are specified in the fields ("Region size (px)"), allowing for any rectangular size. Then, the user decides how ROIs will be placed. If manual placement is required, the "Manual Options" tools will be exploited. By using these tools, the user can place a drag-and-drop rectangle and add a ROI of its location to the list on top. The user can also remove all or selected regions.

If a large number of ROIs must be selected, placement by hand might 
not be practical. A semi-automatic approach is available and implemented through the tools located in the "Automatic Options" section (Fig. 5, area 4) and the "Brush" tool. By toggling the "Edit Mask" button, the user enters a mode for specifying the image mask. This mask is used to guide the automatic ROI placement. In this section, the tools offer the following functionality - creating an initial mask based on a selected threshold value; inverting the mask; growing and shrinking the mask with a specified step; add or remove areas by specifying rectangular and polygonal areas by hand; adding and removing areas with a "Brush" tool. When the desired mask is created, the user then selects the method (and number, if available) of the ROIs with the drop-down menu on the bottom. The toolbox offers ROI selection both in a grid and random patterns. The grid method allows for adjacent placement and for specified overlap (by percentage or pixel overlap). In order to use the random placement, the user specifies the number of ROIs to be used for image population.

\section{- Using the tool}

By means of the toolbox, an image evaluation / analysis is commonly performed following the sequence, presented in the diagram in Fig. 7.

The image of interest is loaded and visualized. The toolbox works with both isotropic and anisotropic images. Part of the computations are invariant to the size of the pixel (e.g. power law, fractal analysis), while for the others, a possibility for resampling to a square pixel is provided. The user defines the parameters of the ROIs (dimensions and locations) to be used for the calculations of the features. ROIs can be created manually or semi-automatically, as discussed in the previous section. Further, the user selects the type(s) of the image analysis and proceeds with the computations. Currently, no option for image pre-processing is provided, however the tool is open and such possibility can be added later, if needed.

Calculations are performed either on a single image or on a batch of images. The batch in the tool is defined as a list of separate images, which may eventually be $2 \mathrm{D}$ slices from a single volume. The batch in scripts may also include stacked images from single files. The parameters of the features are specified in a dedicated .mat file, the content of which is shown in Appendix Part C. The later may be adjusted from the MATLAB console. The source code of the tool (only the customdeveloped scripts, excluding MATLAB-provided toolboxes and core scripts) is open and is available on request together with a complete guide and user supplies.

\subsection{Case studies}

The developed tool was used in a series of image evaluation studies [49-53]. In the following paragraph, we show the use of the tool in two original research applications in the field of x-ray breast imaging: (1) evaluation of the features from a series of left and right (pairs) CC breast clinical mammograms, and (2) evaluation of the suitability of newly proposed and developed breast phantoms for x-ray-based imaging. The first application aimed to obtain reference values of radiomics features for mammograms (free of breast lesions). The second application aimed at evaluation of existing physical breast phantoms by comparing features from their images with reference features from clinical mammography images. Results from such comparison can be used to further improve the phantoms.

- Example 1: Feature extraction in a series of mammographic images

With the newly developed radiomics toolbox we assessed 88 mammography images (left and right CC pairs of each patient, $2 \times 44$ total) selected to be in cranio-caudal (CC) view, with compressed breast thickness ranging from $50 \mathrm{~mm}$ to $59 \mathrm{~mm}$, acquired with Siemens Mammomat Inspiration system (Forchheim, Germany) with a pixel resolution of $85 \mu \mathrm{m} \times 85 \mu \mathrm{m}$. First order statistics, skewness, kurtosis, 


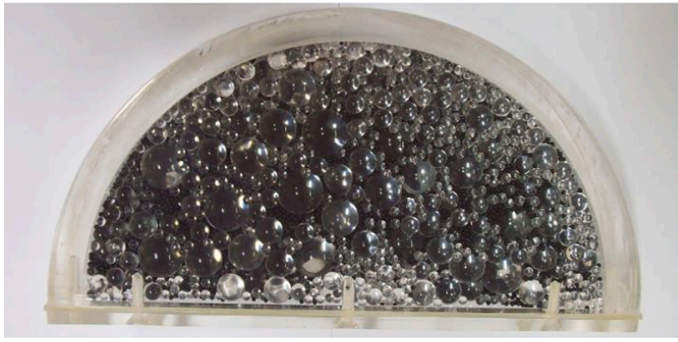

(a)

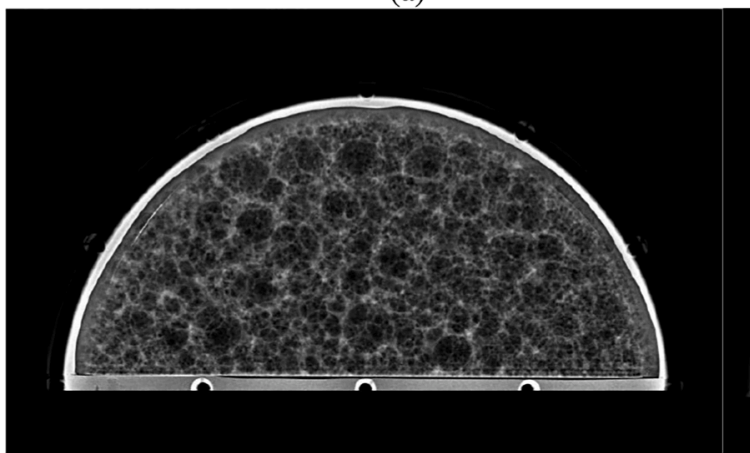

(c)

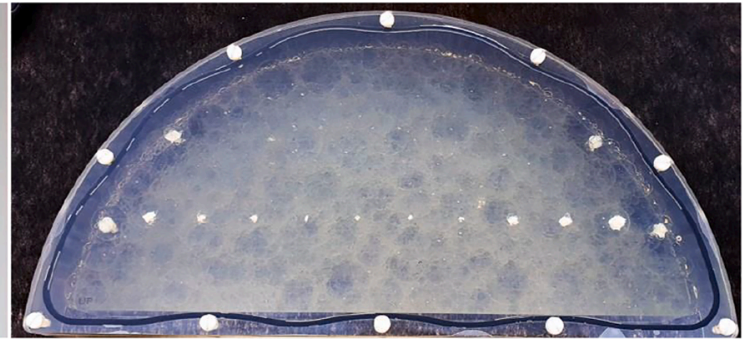

(b)

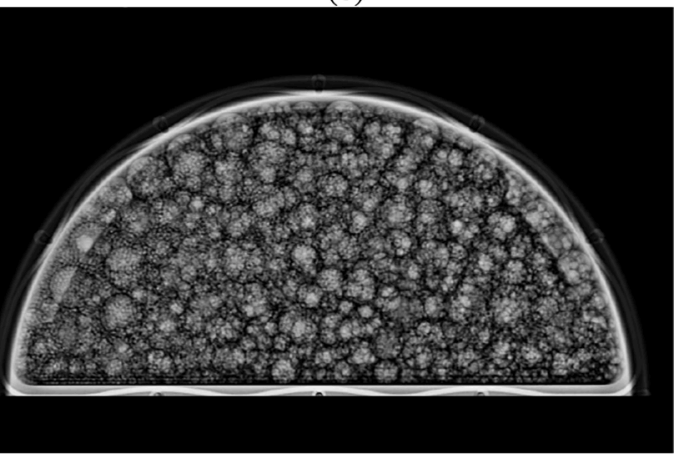

(d)

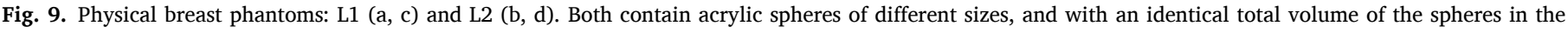
separate size groups. L1 is filled with water, while L2 is filled with paraffin oil.

parameter $\beta$, fractal dimension, GLCM and NGTDM were calculated over at least 100 ROIs in each of the patient mammography images. Next, the Anderson-Darling test was used to determine whether the observed distribution of the individual features from a single image can be considered as normal (Table 1 for left and right mammographic CC images). For the features following the normal distributions, mean values and standard deviations were calculated. Further, histograms of these features were displayed in Fig. 8. The settings used for the calculations were the following: for skewness and kurtosis and other firstorder statistics (minimum, maximum, etc.) the ROI size was 64 pixels $\times 64$ pixels $(5.44 \mathrm{~mm} \times 5.44 \mathrm{~mm}$ ); for the calculation of the power-law exponent $\beta$, fractal dimension, GLCM and NGTDM the ROI size was 128 pixels $\times 128$ pixels $(10.88 \mathrm{~mm} \times 10.88 \mathrm{~mm})$ with $50 \%$ overlap of the regions. All ROIs were placed "inside" the projected breast, excluding skin, muscle and non-breast background, as shown in Figs. 1 and 5.

Table 2 shows the p-values after the null hypothesis testing of features from left and right mammography CC images using $t$-test for features following normal distribution and Mann-Whitney $U$ test for the rest of features.

Measurements for features extracted from the set of 44 pairs of mammograms of left and right breasts demonstrate very close data. Results showed that there is no statistically significant difference (significance level $\alpha=0.05$ ) between the CC view images of our left and right CC mammography datasets. Moreover, the results for skewness of the left and right breast mammography images both demonstrated positive values, suggesting that the mammography images were predominantly from fatty breasts. This is also well supported by the relatively high fractal values (2.7-3.0) and lower $\beta$ values, suggesting that mammograms are from more fatty breasts [44].

- Example 2. Evaluation of newly developed physical phantoms dedicated to x-ray imaging.

This study aimed at evaluating anthropomorphic physical breast phantoms to be used for 3D breast imaging. For this purpose, the radiomics toolbox was used in the evaluation of 100 (left CC or right CC, but not pairs) clinical mammograms. The new phantom ("L2", Fig. 9b) is designed as a successor to the "L1" sphere phantom (Fig. 9a) by a cooperation between the team in KU Leuven and in the University of Vienna [54]. This new iteration has several differences when compared to the first version of the object - L1 has compressed breast thickness equivalent of $6 \mathrm{~cm}$ and physical thickness of $4.8 \mathrm{~cm}$, while L2 has thickness equivalent of $5.5 \mathrm{~cm}$ and physical thickness of $5.35 \mathrm{~cm}$. The spheres in the container have the same diameters and are filled following the same fractal ratios. The L2 phantom has a central plate covering the whole area and has its mass models 3D printed on it, while in the L1, the central plate is a strip, covering part of the whole container and its mass models were glued to the plate. Lastly, the filling between the spheres in the L2 is paraffin oil compared to the distilled water used in L1. Shaking both phantoms rearranges the spheres, thus creating new textures.

Using the radiomics toolbox, we computed the features of the images of the two phantoms (L1 and L2) and we compared them to the reference data of the 100 single images. All images had a pixel pitch of $85 \mu \mathrm{m}$ and were acquired on the same Siemens Inspiration mammography system (Forchheim, Germany), at a tube voltage of $28 \mathrm{kVp}$ under automatic exposure control. For the calculations of the first order statistics, the size of the ROI was $64 \times 64$ pixels, while for the power spectrum analysis and the rest of the features this size was $128 \times 128$ pixels with $50 \%$ overlap. Fig. 10 presents the radiomics feature histograms for the patient and phantom groups.

We followed the approach of Acciavatti et al. [55] and verified whether a given feature is realistic in comparison to features extracted from patient images. More specifically, if this feature is between 2.5th and 97.5th percentiles of the clinical distribution, then we accept that this is a realistic feature. The results are summarised in Table 3 , showing important parameter coincidence for $\beta$, GLCM energy and GLCM homogeneity with clinical data. They are shown in literature to be potentially improving the discrimination power of risk-prediction models [56].

The L1 phantom texture gives $\beta$, fractal dimension, GLCM correlation and NGTDM busyness features that are close to the mean of the distribution of the patient texture features. Only for the GLCM energy and NGDTM Contrast features the values fall out of the range of the patient values. In the case of $\mathrm{L} 2$, there is a close match for the $\beta$ parameter between phantom and patient images (Fig. 10, $\beta$ values). The other 

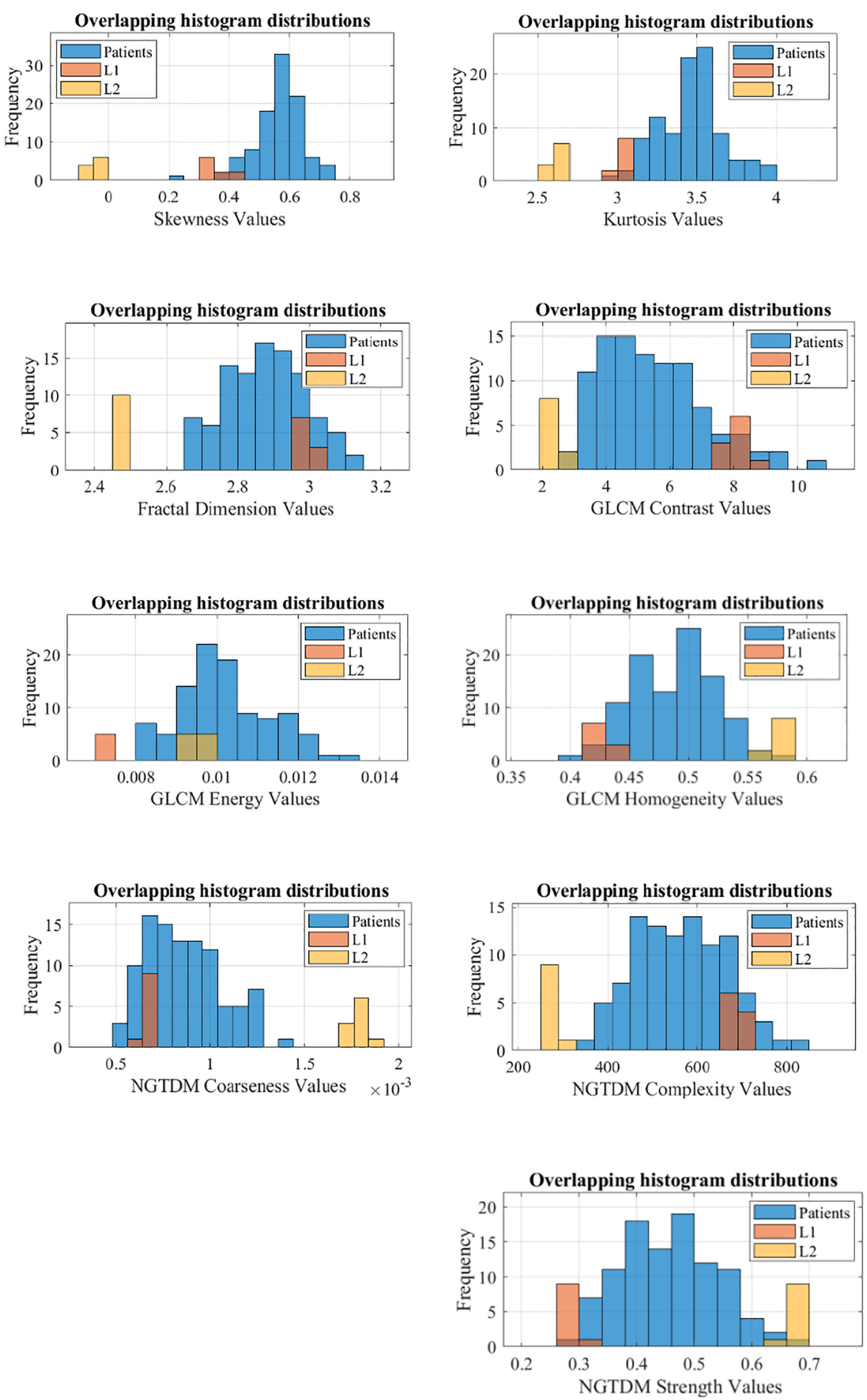
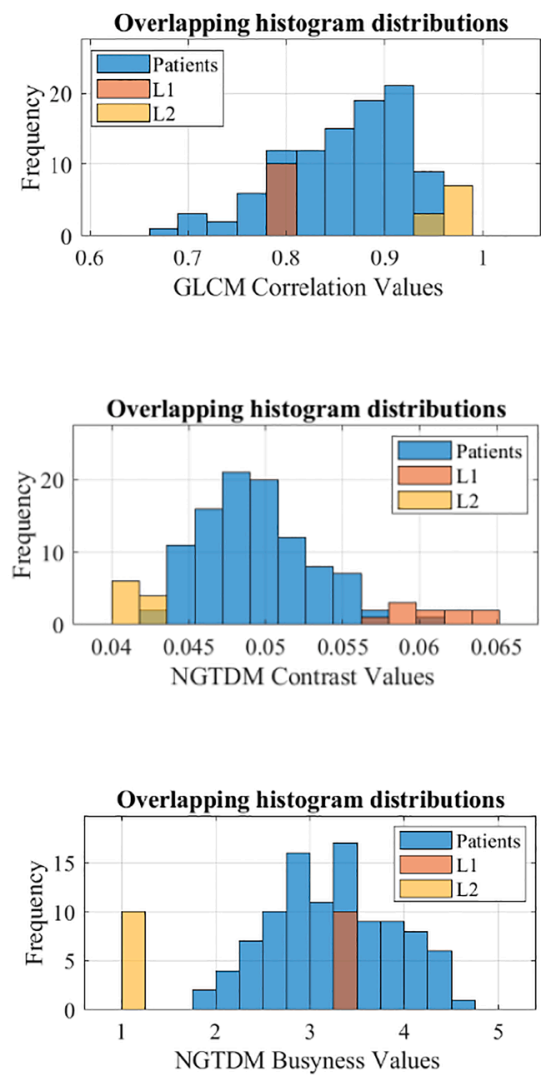

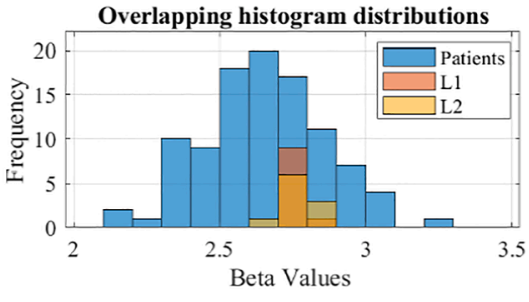

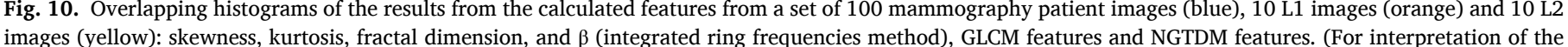
references to colour in this figure legend, the reader is referred to the web version of this article.) 
Table 3

Evaluation of feature realism for L1 and L2 phantoms. $\sqrt{ }$ - realistic, $\times$ - nonrealistic.

\begin{tabular}{lcc}
\hline Feature & L1 & L2 \\
\hline Skewness & $\sqrt{ }$ & $\times$ \\
Kurtosis & $\sqrt{ }$ & $\times$ \\
Beta, $\beta$ & $\sqrt{ }$ & $\sqrt{ }$ \\
Fractal dimension & $\sqrt{ }$ & $\times$ \\
GLCM Contrast & $\sqrt{ }$ & $\times$ \\
GLCM Correlation & $\sqrt{ }$ & $\times$ \\
GLCM Energy & $\times$ & $\sqrt{ }$ \\
GLCM Homogeneity & $\sqrt{ }$ & $\sqrt{ }$ \\
NGDTM Coarseness & $\sqrt{ }$ & $\times$ \\
NGDTM Contrast & $\times$ & $\times$ \\
NGDTM Busyness & $\sqrt{ }$ & $\times$ \\
NGDTM Complexity & $\sqrt{ }$ & $\times$ \\
NGDTM Strength & $\sqrt{ }$ & $\sqrt{ }$ \\
\hline
\end{tabular}

calculated parameters for the L2 phantom differed from the corresponding values from patient data even though the phantom filling material of the L2 phantom, namely acrylic and paraffin oil, are closer in $\mathrm{x}$-ray attenuation to breast glandular and adipose tissue respectively when compared to the material in L1, acrylic and distilled water. The latter results in a model which produces mammograms of more dense breasts. This experiment indicates that radiomics analysis may be powerful tool in the process of improving the physical phantoms. From this first analysis, the L2 phantom seemed inferior to the L1 phantom for the texture analysis characteristics. For the improvement of the characteristics of L2 phantom, the analysis of the mammography images shown in Fig. 10 will be explored.

The developed toolbox turns to be useful in analysing mammography images and in comparing results to outcomes from other clinical and phantom studies. It can help in feature extraction not only from mammographic but also from other types of medical images, provided they fulfil the format requirements specified in section 3.1. The toolbox can be run in MATLAB version 2013a or later. Most of the scripts can be run even in OCTAVE, which is freely available under the terms of the
GNU General Public License. The toolbox provides functionality encapsulated with a user interface, without the need of writing programming code. This is one of the strengths of this tool. The tool is suitable for researchers using MATLAB and can be exploited in any other educational and research activities [61]. It needs rather a short time investment related to the installation of MATLAB, and no time in setting up the tool.

\section{Conclusions}

Using MATLAB, we created a toolbox, including a GUI application for extraction of features of interest, dedicated for applications in breast imaging modalities. The toolbox was successfully used for two practical questions in actual mammography research. The tool was designed for applications in breast imaging, but it is not limited to this application as other imaging modalities use similar techniques. In addition, the tool is available to the community upon reasonable request and its functionality can also be easily expanded to suit more specific needs and applications.

\section{Declaration of Competing Interest}

The authors declare that they have no known competing financial interests or personal relationships that could have appeared to influence the work reported in this paper.

\section{Acknowledgments}

This work was supported by the Bulgarian National Science Fund under grant agreement [DN17/2]. The authors also wish to acknowledge financial support from the FFG-FWO, [Grant No. 861552], between the University of Vienna and the KU Leuven. The authors would like to thank Elisabeth Salomon from University of Vienna for providing the L2 phantom for this study.

\section{Appendix}

\section{A. Coded features}

\begin{tabular}{|c|c|}
\hline Feature & Calculation \\
\hline Skewness & $\begin{array}{l}\text { Skewness }=\sum \frac{\left(X_{i}-\bar{X}\right)^{3}}{n \sigma^{3}} \text { (A1) } \\
X_{i} \text { stands for the individual pixel intensity values of the ROI, } \bar{X} \text { is their mean value, } \sigma \text { is the standard deviation, and } n \text { is the sample size (i.e., the number of the } \\
\text { pixels in the ROI). The tool also computes the unbiased value for the skewness [37], by substituting } n \text { in equation } 3 \text { with }(n-1) \text {. }\end{array}$ \\
\hline Kurtosis & $\begin{array}{l}\text { Kurtosis }=\sum \frac{\left(X_{i}-\bar{X}\right)^{4}}{n \sigma^{4}}(\mathrm{~A} 2) \\
\text { The unbiased value is also calculated. }\end{array}$ \\
\hline Fractal dimension & $\begin{array}{l}\text { The current implementation is similar to the "box-counting technique" [26]. If the image pixels are represented in three-dimensional space as adjacent prismatic } \\
\text { bars, the proposed method computes the area } A \text {, of the total surface they form. In case of square pixels of size } \varepsilon \text {, this area is calculated as follows: } A(\varepsilon)=\sum_{x, y} \varepsilon^{2}+ \\
\sum_{x y y} \varepsilon\left(\left|i_{\varepsilon}(x, y)-i_{\varepsilon}(x, y+1)\right|+\left|i_{\varepsilon}(x, y)-i_{\varepsilon}(x+1, y)\right|\right) \\
\text { where } i_{\varepsilon}(x, y) \text { is the pixel value at location }(x, y) \text {. The fractal dimension } D \text { of an image is then calculated as follows: } \\
D=2-\frac{\Delta \log [A(\varepsilon)]}{\Delta \log [\varepsilon]} \text { (A3) } \\
\text { and the resulting value reported by the tool is the average over all the ROIs. }\end{array}$ \\
\hline Power law & $\begin{array}{l}\text { For mammographic images, the magnitude of the low-frequency components shows a power law behaviour }[41] \text { : } \\
P(f)=\frac{c}{f^{\beta}}(\mathrm{A} 4) \\
\text { where } P \text { is the power spectrum, } c \text { is a constant, } f \text { represents the spatial frequency }(\mathrm{lp} / \mathrm{mm}) \text { in the radial direction, and } \beta \text { is a factor that determines the slope of the } \\
\lg (P) \text { vs. } \lg (f) \text { graph. The descriptive parameter } \beta \text { has been used to characterize the texture of the breast. }\end{array}$ \\
\hline GLCM Energy & $\begin{array}{l}\mathrm{F}_{\text {GLCMEnergy }}=\sum_{i=1}^{N_{g}} \sum_{j=1}^{N_{g}} p_{i j}^{2} \text { (A5) } \\
\text { where } N_{g} \text { is the number of discretized grey levels present in the ROI and } \mathrm{p}_{\mathrm{ij}} \text { is the probability value for the ij-th grey tone from the calculated GLCM matrix. }\end{array}$ \\
\hline $\begin{array}{r}\text { The GLCM } \\
\text { Contrast }\end{array}$ & $\begin{array}{l}\mathrm{F}_{G L C M C o n t r a s t}=\sum_{i=1}^{N_{g}} \sum_{j=1}^{N_{g}}(i-j)^{2} p_{i j}(\mathrm{~A} 6) \\
\text { For a constant image, the GLCM Contrast is } 0 .\end{array}$ \\
\hline
\end{tabular}


(continued)

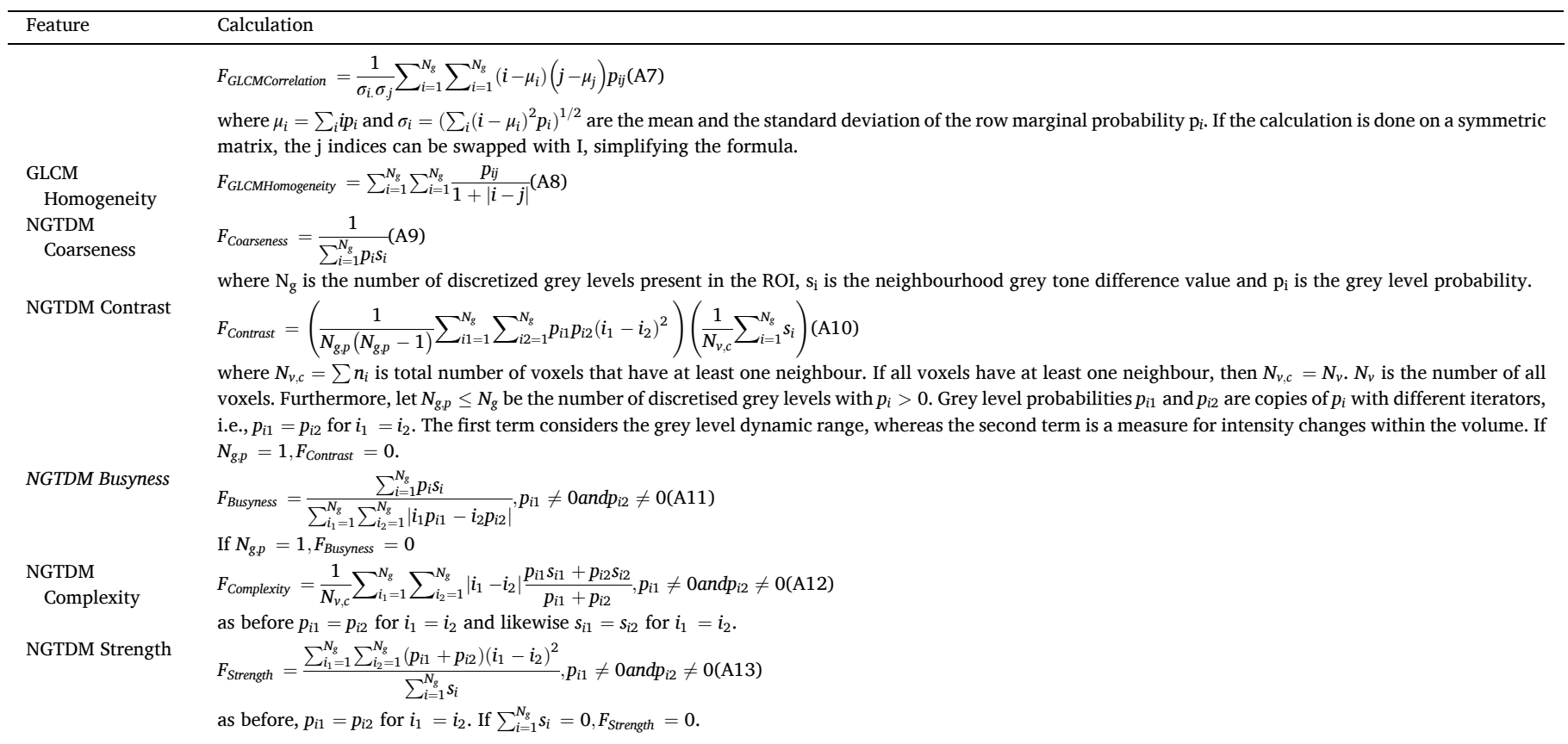

\section{B. Feature Verification}

The verification of the toolbox features was performed with both the pyRadiomics library [35]. The pyRadiomics library is based on the feature definitions described in the IBSI document. For validation purposes, two types of phantoms are used: the digital phantom described in the IBSI document (chapter 5.1, IBSI [36]), and patient clinical 2D and 3D mammography images. The digital phantom is a matrix with size of $5 \times 4 \times 4$ pixels and is presented in MATLAB as follows:

IBSIphantom = zeros $(4,5,4) ; \%$ [Row, Col, Depth]

IBSIphantom(:::,1) $=[1,4,4,1,1 ; 1,4,6,1,1 ; 4,1,6,4,1 ; 4,4,6,4,1]$;

IBSIphantom(:,:,2) $=[1,4,4,1,1 ; 1,1,6,1,1 ; 1,1,3,1,1 ; 4,4,6,1,1]$;

IBSIphantom(:,:,3) $=[1,4,4,1,1 ; 1,1,1,1,1 ; 1,1,9,1,1 ; 1,1,6,1,1]$;

IBSIphantom(:,:,4) $=[1,4,4,1,1 ; 1,1,1,1,1 ; 1,1,1,1,1 ; 1,1,6,1,1]$;

Table 4 lists the calculated features. Results showed an excellent match between calculated features.

Patient clinical 2D and 3D mammography images were selected randomly from two publicly available datasets with clinical mammography images: the Maxima database [57] and a new dataset of breast phantom images registered at Zenodo public repository [58], both being at the basis for the development of 3D physical and computational breast phantoms [59] and virtual clinical trials in breast imaging [60]. Fig. 11a, b shows ROIs

Table 4

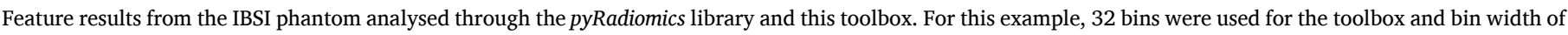
20.84 was used for the pyRadiomics library for the rescaling. Step and neighborhood for the GLCM and NGTDM were set to 1.

\begin{tabular}{|c|c|c|}
\hline & pyRadiomics & This toolbox \\
\hline energy & 567.00 & 567.00 \\
\hline kurtosis & 2.65 & 2.65 \\
\hline $\max$ & 6.00 & 6.00 \\
\hline mean & 2.15 & 2.15 \\
\hline median & 1.00 & 1.00 \\
\hline $\min$ & 1.00 & 1.00 \\
\hline range & 5.00 & 5.00 \\
\hline variance & 1.08 & 1.08 \\
\hline skewness & 3.05 & 3.05 \\
\hline GLCM Contrast & 5.32 & 5.32 \\
\hline GLCM Correlation & 0.16 & 0.16 \\
\hline GLCM Homogeneity & 0.68 & 0.68 \\
\hline GLCM Energy & 0.30 & 0.30 \\
\hline NGTDM Busyness & 6.54 & 6.54 \\
\hline NGTDM Coarseness & 0.03 & 0.03 \\
\hline NGTDM Complexity & 13.54 & 13.54 \\
\hline NGTDM Contrast & 0.58 & 0.58 \\
\hline NGTDM Strength & 0.76 & 0.76 \\
\hline
\end{tabular}




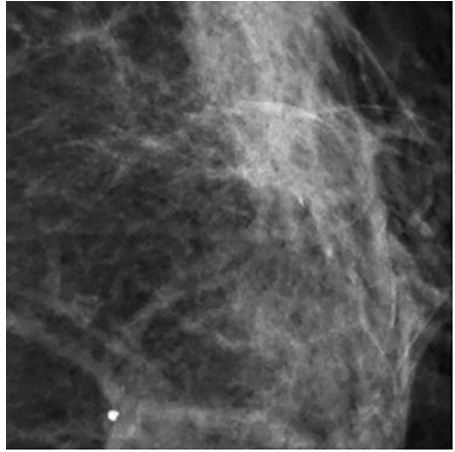

a)

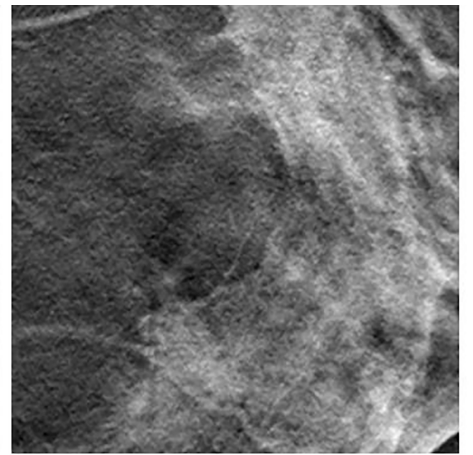

b)

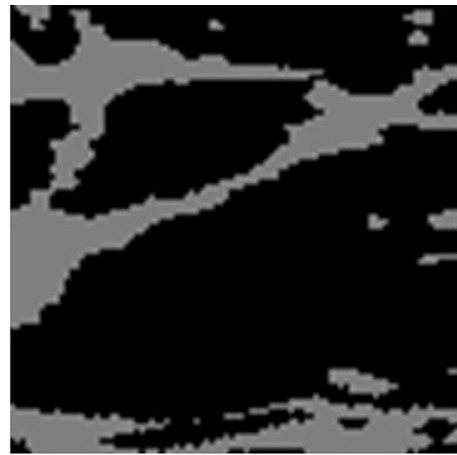

c)

Fig. 11. Region of interests (ROIs) used for validation of features: ROI extracted from a patient (a) mammography image, (b) slice from a reconstructed tomosynthesis volume and (c) phantom CT slice. ROIs in (a) and (b) have dimensions of $500 \times 500$ pixels, while in (c) the ROI is $90 \times 90$ pixels.

Table 5

Comparison of features extracted from publicly available clinical images [58,59] by using the pyRadiomics library and this toolbox. The first pair of columns (a) lists the features extracted from mammography ROI, the second (b) is from the central slice of a reconstructed tomosynthesis volume (Fig. 11 b) and the last (c) from an isometric phantom voxel model. For this example, 32 bins were used for both pyRadiomics and the toolbox and for the rescaling. Step and neighbourhood parameters for GLCM and NGTDM were set to 1 .

\begin{tabular}{|c|c|c|c|c|c|c|}
\hline & \multicolumn{2}{|c|}{ Patient mammographic image (a) } & \multicolumn{2}{|c|}{ Patient tomosynthesis (b) } & \multicolumn{2}{|c|}{ Segmented Phantom Slice (c) } \\
\hline & pyRadiomics & MATLAB & pyRadiomics & MATLAB & pyRadiomics & MATLAB \\
\hline Maximum & 4004.00 & 4004.00 & 2679.00 & 2679.00 & 2.00 & 2.00 \\
\hline Minimum & 822.00 & 822.00 & 1505.00 & 1505.00 & 1.00 & 1.00 \\
\hline Range & 3182.00 & 3182.00 & 1174.00 & 1174.00 & 1.00 & 1.00 \\
\hline Median & 1707.00 & 1707.00 & 1957.00 & 1957.00 & 1.00 & 1.00 \\
\hline Mean & 1804.49 & 1804.50 & 1985.52 & 1985.50 & 1.25 & 1.25 \\
\hline Variance & $2.22 \mathrm{E}+05$ & $2.22 \mathrm{E}+05$ & $3.53 \mathrm{E}+04$ & $3.53 \mathrm{E}+04$ & 0.19 & 0.19 \\
\hline Skewness & 0.72 & 0.72 & 0.43 & 0.43 & 1.14 & 1.14 \\
\hline Kurtosis & 3.01 & 3.01 & 2.44 & 2.44 & 2.30 & 2.30 \\
\hline Energy & $8.63 \mathrm{E}+11$ & $8.63 E+11$ & $9.86 \mathrm{E}+11$ & $9.86 \mathrm{E}+11$ & $1.36 \mathrm{E}+04$ & $1.36 \mathrm{E}+04$ \\
\hline GLCM Contrast & 1.34 & 1.34 & 1.72 & 1.72 & 70.36 & 70.36 \\
\hline GLCM Correlation & 0.97 & 0.97 & 0.97 & 0.97 & 0.80 & 0.80 \\
\hline GLCM Energy & $1.74 \mathrm{E}-02$ & $1.74 \mathrm{E}-02$ & $1.48 \mathrm{E}-02$ & $1.48 \mathrm{E}-02$ & $5.58 \mathrm{E}-01$ & 5.58E-01 \\
\hline GLCM Homogeneity & 0.65 & 0.65 & 0.66 & 0.66 & 0.93 & 0.93 \\
\hline NGTDM Coarseness & $1.06 \mathrm{E}-04$ & $1.06 \mathrm{E}-04$ & $1.42 \mathrm{E}-04$ & $1.42 \mathrm{E}-04$ & $1.13 \mathrm{E}-04$ & $1.13 \mathrm{E}-04$ \\
\hline NGTDM Contrast & $2.76 \mathrm{E}-02$ & $2.76 \mathrm{E}-02$ & $2.76 \mathrm{E}-02$ & $2.76 \mathrm{E}-02$ & $4.13 \mathrm{E}+02$ & $4.13 \mathrm{E}+02$ \\
\hline NGTDM Busyness & 28.46 & 28.46 & 17.34 & 17.34 & 602.65 & 602.65 \\
\hline NGTDM Complexity & 308.33 & 308.33 & 233.10 & 233.10 & 70.62 & 70.62 \\
\hline NGTDM Strength & $6.16 \mathrm{E}-02$ & $6.16 \mathrm{E}-02$ & $5.95 \mathrm{E}-02$ & $5.95 \mathrm{E}-02$ & 0.11 & 0.11 \\
\hline
\end{tabular}

extracted from a mammographic image (Maxima database) and a tomosynthesis slice of an imaged patient breast (Maxima database), while Fig. 11c shows a ROI from a phantom CT slice from the new dataset of breast phantom images registered at Zenodo. In the latter case, the grey levels correspond to the basic breast tissue types: adipose and glandular tissue. The results, summarised in Table 5, showed an exact match to the measurements performed with the pyRadiomics.

\section{Default feature values}

Default values of the included features are shown in Table 6. These features are stored in a .mat file, which can be loaded in the MATLAB

Table 6

Default feature parameters specified in the dedicated .mat file.

\begin{tabular}{|c|c|c|}
\hline Feature parameter & Value & Comment \\
\hline afBetaLow & 0.1 & Lower frequency for the $\beta$ value \\
\hline afBetaHigh & 1 & Higher frequency for the $\beta$ value \\
\hline fractalIterations & 8 & Number of iterations for the Fractal Dimension \\
\hline GLCMDistance & 1 & Pixel distance for the GLCM feature \\
\hline GLCMGrayLevels & 32 & Number of gray levels to rescale the image to before extracting the features \\
\hline GLCMGrayLimits & 0 & Range used scaling input image into gray levels, specified as a 2-element vector [low high] \\
\hline GLCMSymmetry & 1 & Specify if the resulting matrices with opposite directions are summed \\
\hline NGTDMDistance & 1 & Pixel neighbourhood distance for the NGTDM feature \\
\hline NGTDMGrayLevels & 32 & Number of gray levels to rescale the image to before extracting the features \\
\hline
\end{tabular}


environment.

The content of the mat file can be updated by the following procedure (via the console window):

>load('settings\fParams.mat');

\begin{tabular}{|ll}
\hline -E 1x1 struct with 9 fields & \\
\hline Field 4 & Value \\
$\boxplus$ afBetaLow & 0.1000 \\
$\boxplus$ afBetaHigh & 1 \\
$\boxplus$ fractallterations & 8 \\
$\boxplus$ GLCMDistance & 1 \\
$\boxplus$ GLCMGrayLevels & 32 \\
$\boxplus$ GLCMGrayLimits & 0 \\
$\boxplus$ GLCMSymmetry & 1 \\
$\boxplus$ NGTDMDistance & 1 \\
\hline NGTDMGrayLevels & 32 \\
\hline & \\
>> Save ( ' fParams.mat ' , ' fParams ' );
\end{tabular}

\section{References}

[1] Bliznakova K, Bliznakov Z, Bravou V, Kolitsi Z, Pallikarakis N. A three-dimensional breast software phantom for mammography simulation. Phys Med Biol 2003;48 (22):3699-719.

[2] Bliznakova K, Sechopoulos I, Buliev I, Pallikarakis N. BreastSimulator: a software platform for breast x-ray imaging research. J Biomed Graph Comput 2012;2(1). https://doi.org/10.5430/jbgc.v2n1p1.

[3] Bliznakova K, Suryanarayanan S, Karellas A, Pallikarakis N. Evaluation of an improved algorithm for producing realistic 3D breast software phantoms: application for mammography. Med Phys 2010;37(11):5604-17.

[4] Bliznakova K, Russo P, Mettivier G, Requardt H, Popov P, Bravin A, et al, A software platform for phase contrast x-ray breast imaging research. Comput Biol Med 2015;61:62-74.

[5] Mettivier G, Bliznakova K, Sechopoulos I, Boone JM, Di Lillo F, Sarno A, et al. Evaluation of the BreastSimulator software platform for breast tomography. Phys Med Biol 2017;62(16):6446-66.

[6] MacKenzie A, et al. Comparison of synthetic 2D images with planar and tomosynthesis imaging of the breast using a virtual clinical trial. Progress in Biomedical Optics and Imaging - Proceedings of SPIE. 2018.

[7] Sharma D, Graff CG, Badal A, Zeng R, Sawant P, Sengupta A, et al. Technical Note: In silico imaging tools from the VICTRE clinical trial. Med Phys 2019;46(9): 3924-8.

[8] Badano A, Graff CG, Badal A, Sharma D, Zeng R, Samuelson FW, et al. Evaluation of digital breast tomosynthesis as replacement of full-field digital mammography using an in silico imaging trial. JAMA Netw Open 2018;1(7):e185474. https://doi org/10.1001/jamanetworkopen.2018.5474.

[9] Badano A, et al. In silico imaging clinical trials for regulatory evaluation: Initial considerations for VICTRE, a demonstration study. Progress in Biomedical Optics and Imaging - Proceedings of SPIE. 2017.

[10] Bakic PR, et al. Virtual clinical trial of lesion detection in digital mammography and digital breast tomosynthesis. 2018: SPIE.

[11] Russo P, Mettivier G, Lauria A, Montesi MC. X-ray cone-beam breast computed tomography: phantom studies. IEEE Trans Nucl Sci 2010;57(1):160-72.

[12] Mettivier G, Russo P, Cesarelli M, Ospizio R, Passeggio G, Roscilli L, et al. Dedicated scanner for laboratory investigations on cone-beam CT/SPECT imaging of the breast. Nucl Instrum Methods Phys Res Sect A-Accelerators Spectrometers Detectors Associated Equipment 2011;629(1):350-6.

[13] Sarno A, Mettivier G, Russo P. Dedicated breast computed tomography: Basic aspects. Med Phys 2015;42(6Part1):2786-804.

[14] Sarno A, Mettivier G, Di Lillo F, Cesarelli M, Bifulco P, Russo P. Cone-beam micro computed tomography dedicated to the breast. Med Eng Phys 2016;38(12): $1449-57$.

[15] Kalender WA, Beister M, Boone JM, Kolditz D, Vollmar SV, Weigel MCC. Highresolution spiral CT of the breast at very low dose: concept and feasibility considerations. Eur Radiol 2012;22(1):1-8.

[16] Boone JM, Nelson TR, Lindfors KK, Seibert JA. Dedicated breast CT: radiation dose and image quality evaluation. Radiology 2001;221(3):657-67.

[17] Sarno A, Mettivier G, Golosio B, Oliva P, Spandre G, Di Lillo F, et al. Imaging performance of phase-contrast breast computed tomography with synchrotron radiation and a CdTe photon-counting detector. Phys Med 2016;32(5):681-90.

[18] Longo R, Arfelli F, Bellazzini R, Bottigli U, Brez A, Brun F, et al. Towards breast tomography with synchrotron radiation at Elettra: first images. Phys Med Biol 2016;61(4):1634-49.

[19] Delogu P, Golosio B, Fedon C, Arfelli F, Bellazzini R, Brez A, et al. Imaging study of a phase-sensitive breast-CT system in continuous acquisition mode. J Instrum 2017;12(01):C01016.
[20] Ding H, Ducote JL, Molloi S. Measurement of breast tissue composition with dual energy cone-beam computed tomography: a postmortem study. Med Phys 2013;40 (6Part1):061902. https://doi.org/10.1118/1.4802734.

[21] Heine JJ, Velthuizen RP. Spectral analysis of full field digital mammography data. Med Phys 2002;29(5):647-61.

[22] Abbey CK, Nosratieh A, Sohl-Dickstein J, Yang K, Boone JM. Non-Gaussian statistical properties of breast images. Med Phys 2012;39(11):7121-30.

[23] Mainprize JG, Tyson AH, Yaffe MJ. The relationship between anatomic noise and volumetric breast density for digital mammography. Med Phys 2012;39(8):4660-8.

[24] Cockmartin L, et al. Power spectrum analysis of an anthropomorphic breast phantom compared to patient data in 2D digital mammography and breast tomosynthesis. In: Lecture Notes in Computer Science (including subseries Lecture Notes in Artificial Intelligence and Lecture Notes in Bioinformatics); 2014. p. 423-9.

[25] Lopes R, Betrouni N. Fractal and multifractal analysis: a review. Med Image Anal 2009; 13(4):634-49.

[26] Byng JW, Boyd NF, Fishell E, Jong RA, Yaffe MJ. Automated analysis of mammographic densities. Phys Med Biol 1996;41(5):909-23.

[27] Karssemeijer N, Hendriks JHCL. Computer-assisted reading of mammograms. Eur Radiol 1997;7(5):743-8.

[28] Shepherd JA, Herve L, Landau J, Fan B, Kerlikowske K, Cummings SR. Clinical comparison of a novel breast DXA technique to mammographic density. Med Phys 2006;33(5):1490-8.

[29] Conroy SM, Woolcott CG, Koga KR, Byrne C, Nagata C, Ursin G, et al Mammographic density and risk of breast cancer by adiposity: an analysis of four case-control studies. Int J Cancer 2012;130(8):1915-24.

[30] Tagliafico A, Tagliafico G, Astengo D, Airaldi S, Calabrese M, Houssami N. Comparative estimation of percentage breast tissue density for digital mammography, digital breast tomosynthesis, and magnetic resonance imaging. Breast Cancer Res Treat 2013;138(1):311-7.

[31] Boyd NF, Lockwood GA, Martin LJ, Knight JA, Byng JW, Yaffe MJ, et al. Mammographic densities and breast cancer risk. Cancer Epidemiol Biomarkers Prev 1998;10(3-4):113-26.

[32] McCormack VA, Dos Santos Silva I. Breast density and parenchymal patterns as markers of breast cancer risk: a meta-analysis. Cancer Epidemiol Biomarkers Prev 2006;15(6):1159-69.

[33] Alonzo-Proulx O, Jong RA, Yaffe MJ. Volumetric breast density characteristics as determined from digital mammograms. Phys Med Biol 2012;57(22):7443-57.

[34] Nazari SS, Mukherjee P. An overview of mammographic density and its association with breast cancer. Breast Cancer 2018;25(3):259-67.

[35] van Griethuysen JJM, Fedorov A, Parmar C, Hosny A, Aucoin N, Narayan V, et al. Computational radiomics system to decode the radiographic phenotype. Cancer Res 2017;77(21):e104-7.

[36] Zwanenburg A, et al. Image biomarker standardisation initiative arXiv e-prints, 2016. arXiv:1612.07003.

[37] Bochud F, Abbey C, Eckstein M. Statistical texture synthesis of mammographic images with super-blob lumpy backgrounds. Opt Express 1999;4(1):33-42.

[38] Caldwell CB, Stapleton SJ, Holdsworth DW, Jong RA, Weiser WJ, Cooke G, et al. Characterisation of mammographic parenchymal pattern by fractal dimension. Phys Med Biol 1990;35(2):235-47.

[39] Li H, Giger ML, Olopade OI, Lan Li. Fractal analysis of mammographic parenchymal patterns in breast cancer risk assessment. Acad Radiol 2007;14(5): 513-21.

[40] Li H, et al. Computerized texture analysis of mammographic parenchymal patterns of digitized mammograms. Acad Radiol 2005;12(7):863-73.

[41] Heine JJ, Deans SR, Velthuizen RP, Clarke LP. On the statistical nature of mammograms. Med Phys 1999;26(11):2254-65. 
[42] Haralick RM, Shanmugam K, Dinstein I. Textural features for image classification. IEEE Trans Syst Man Cybern 1973;SMC-3(6):610-21.

[43] Castella C, Abbey CK, Eckstein MP, Verdun FR, Kinkel K, Bochud FO. Human linear template with mammographic backgrounds estimated with a genetic algorithm. J Opt Soc Am A Opt Image Sci Vis 2007;24(12):B1. https://doi.org/10.1364/ JOSAA.24.0000B1.

[44] Li H, Giger ML, Olopade OI, Chinander MR. Power spectral analysis of mammographic parenchymal patterns for breast cancer risk assessment. J Digit Imaging 2008;21(2):145-52.

[45] Kontos D, Bakic PR, Carton A-K, Troxel AB, Conant EF, Maidment ADA. Parenchymal texture analysis in digital breast tomosynthesis for breast cancer risk estimation: a preliminary study. Acad Radiol 2009;16(3):283-98.

[46] Amadasun M, King R. Textural features corresponding to textural properties. IEEE Trans Syst Man Cybern 1989;19(5):1264-74.

[47] Marshall NW. A comparison between objective and subjective image quality measurements for a full field digital mammography system. Phys Med Biol 2006;51 (10):2441-63.

[48] MathWorks. Matlab-MathWorks. 2016; Available from: https://www.mathworks. com/products/matlab.html.

[49] Baneva Y, Bliznakova K, Cockmartin L, Marinov S, Buliev I, Mettivier G, et al. Evaluation of a breast software model for 2D and 3D X-ray imaging studies of the breast. Phys Med 2017;41:78-86.

[50] Gospodinova G, Bliznakova K. An approach of modelling of breast lesions, in Proceedings of the 1st International Conference "Applied Computer Technologies". Ohrid, Macedonia: Ohrid: University of Information Science and Technology "St. Paul the Apostle" -; 2018. p. 149-53.

[51] Feradov F, Marinov S, Bliznakova K. Physical Breast Phantom Dedicated for Mammography Studies. In: Henriques J, Neves N, de Carvalho P, editors. XV Mediterranean Conference on Medical and Biological Engineering and Computing - MEDICON 2019. MEDICON 2019. IFMBE Proceedings, 2020. vol 76. Springer, Cham. https://doi.org/10.1007/978-3-030-31635-8_41.
[52] Bliznakova K, et al. Validation of a software platform for 2D and 3D phase contrast imaging: Preliminary subjective evaluation. Proceedings of SPIE - The International Society for Optical Engineering. 2020.

[53] Dukov NT, et al. An approach for printing tissue-mimicking abnormalities dedicated to applications in breast imaging. In: 28th International Scientific Conference Electronics, ET 2019 - Proceedings 2019.

[54] Salomon E, et al. Equivalent breast thickness and dose sensitivity of a next iteration 3D structured breast phantom with lesion models. In: Medical Imaging 2020: Physics of Medical Imaging. 2020: SPIE.

[55] Acciavatti RJ, et al. Calculation of radiomic features to validate the textural realism of physical anthropomorphic phantoms for digital mammography. 2020: SPIE; 2020.

[56] Malkov S, Shepherd JA, Scott CG, Tamimi RM, Ma L, Bertrand KA, et al. Mammographic texture and risk of breast cancer by tumor type and estrogen receptor status. Breast Cancer Res 2016;18(1). https://doi.org/10.1186/s13058016-0778-1.

[57] Bliznakova K, Dukov N, Feradov F, Gospodinova G, Bliznakov Z, Russo P, et al. Development of breast lesions models database. Phys Med 2019;64:293-303.

[58] Sarno A, Mettivier G, Franco F, Varallo A, Bliznakova K, Hernandez AM, et al. Dataset of patient-derived digital breast phantoms for in silico studies in breast computed tomography, digital breast tomosynthesis, and digital mammography. Med Phys 2021;48(5):2682-93. https://doi.org/10.1002/mp.v48.510.1002/ mp.14826.

[59] Bliznakova Kristina. The advent of anthropomorphic three-dimensional breast phantoms for X-ray imaging. Phys Med 2020;79:145-61.

[60] di Franco F, Sarno A, Mettivier G, Hernandez AM, Bliznakova K, Boone JM, et al. GEANT4 Monte Carlo simulations for virtual clinical trials in breast X-ray imaging: Proof of concept. Phys Med 2020;74:133-42.

[61] Bliznakova K, Buliev I, Bliznakov Z. Anthropomorphic Phantoms in Image Quality and Patient Dose Optimization. In: A EUTEMPE Network book. 2018, IOP Publishing, online ISBN: 978-0-7503-1323-0, Print ISBN: 978-0-7503-1324-7. 